\title{
Epidemiological and molecular surveillance of influenza and respiratory syncytial viruses in children with acute respiratory infections (2004/2005 season)
}

\author{
Alessandra Zappa', Silvana Perin³, Antonella Amendola', Silvia Bianchi', Elena Pariani', \\ Maria Lorena Ruzza ${ }^{4}$, Alberto Podestà ${ }^{4}$, Elisabetta Tanzi ${ }^{1,2}$, Claudio Farina ${ }^{3}$ \\ 'Dipartimento di Sanità Pubblica-Microbiologia-Virologia, sezione Virologia Applicata alla Sanità Pubblica, Università degli \\ Studi di Milano \\ ${ }^{2}$ CIRI-IV, Centro Interuniversitario di Ricerca sull'Influenza e sulle Infezioni Virali \\ ${ }^{3}$ UOC Microbiologia, AO Ospedale S. Carlo Borromeo, Milano \\ ${ }^{4}$ UOC Pediatria, AO Ospedale S. Carlo Borromeo, Milano
}

Key words: Epidemiological surveillance, virological surveillance, influenza virus, RSV, ARI

Indagine epidemiologico-molecolare dei virus influenzali e respiratorio sinciziale (stagione 20042005) in bambini con infezioni respiratorie acute

\section{SUMMARY}

Objective. During the 2004/2005 influenza season an active virological surveillance of influenza viruses and respiratory syncytial virus (RSV) was carried out to monitor the epidemiologic trend of acute respiratory infections (ARI) in the paediatric community.

Materials and methods. 100 patients (5I males, 49 females; mean age: 19 months), either treated at the Emergency Unit or hospitalized in the Pediatric Unit of "San Carlo Borromeo Hospital" (Milan), reporting symptoms related to ARI were enrolled. Pharyngeal swabs were collected for virological investigation by: I) multiplexnested-PCR for the simultaneous identification of both influenza $A$ and $B$ viruses and RSV; 2 ) multiplex-nestedPCR for the subtyping of influenza $A$ viruses ( $\mathrm{HI}$ and $\mathrm{H} 3$ ).

Results. $12 \%$ (12/100) subjects were infected with influenza $A$ virus, $4 \%(4 / 100)$ with influenza $B$ virus and 14 (14\%) with RSV. Of all the 12 influenza A positive samples 4 (33.3\%) belonged to subtype $\mathrm{HI}$ and 8 (66.7\%) to subtype $\mathrm{H} 3$. Bronchiolitis and bronchitis episodes were significantly higher among RSV-infected subjects than among influenza-infected subjects ( $42.8 \%$ vs $6.2 \%$; $\mathrm{p}<0.05$ and $35.7 \%$ vs $6.2 \%$; $\mathrm{p}<0.05$, respectively). Pneumonia episodes occurred similarly both in influenza-infected children and in RSV-infected ones.

Conclusions. During the 2004/2005 influenza season, influenza viruses and RSV were liable for high morbidity among paediatric subjects. The present study underlies the importance of planning an active surveillance of respiratory viral infections among paediatric cases requiring hospitalization due to ARI.A thorough analysis of target population features, of viruses antigenic properties and seasonality will be decisive in the evaluation of each clinical event.

\section{INTRODUZIONE}

Le ARI (Acute Respiratory tract Infections) costituiscono un problema sanitario di grande rilevanza, poiché sono una delle principali cause di morbosità e mortalità a livello mondiale (7).

Sono infezioni ubiquitarie, altamente contagiose, trasmissibili per via aerea che colpiscono soggetti di diverse età. Inoltre si possono presentare più volte nell'arco della vita in quanto determinate da una molteplicità di microrganismi. I virus sono di gran lunga gli agenti patogeni più comunemente in causa: attualmente sono noti più di 200 sierotipi, antigenicamente differenti, correlati ad infezioni respiratorie $(7,9,15)$.

In base al distretto interessato, alte o basse vie aeree, le infezioni respiratorie possono differenziarsi in URI (Upper Respiratory tract Infections), quali raffreddore, faringite, laringite, tracheite e in LRI (Lower Respiratory tract Infections), come bronchite, bronchiolite e polmonite (9-15).

Nelle fascia di età pediatrica, le infezioni respiratorie acute rappresentano una patologia molto comu-

\section{Corresponding author: Elisabetta Tanzi}

Dipartimento di Sanità Pubblica-Microbiologia-Virologia - Università degli Studi di Milano - Via Pascal 38, 20133 Milano Tel 02 503।5139 - Fax 02 503।5I20 - E-mail: elisabetta.tanzi@unimi.it 
ne e diffusa, frequentemente di natura virale. Le infezioni più comuni riguardano le alte vie respiratorie, mentre le LRI, pur essendo meno frequenti, causano quadri clinici più severi con maggiori rischi di complicanze ed ospedalizzazioni. Ė stato stimato che circa un terzo dei bambini sviluppa un'infezione virale del basso tratto respiratorio entro il primo anno di vita (9). A causa dell'elevata probabilità di reinfezioni, nonchè di sovrainfezioni batteriche, il rischio di complicanze in questa fascia d'età risulta molto elevato. La percentuale di LRI diminuisce fino al $5-10 \%$ nei bambini in età scolare, mentre negli adulti risulta inferiore al 5\%, per poi aumentare di nuovo negli anziani (13).

Dato l'ampio spettro di sintomi comuni causati da molti agenti eziologici responsabili di ARI, non è possibile effettuare una diagnosi precisa basandosi solamente sulla sintomatologia. È noto, infatti, come lo stesso agente possa causare quadri clinici diversi e come il medesimo quadro possa essere attribuito a differenti agenti. Per tale motivo la precisa definizione del ruolo eziologico può avvenire solo tramite indagini di tipo virologico-molecolare (8).

Nei bambini di età inferiore ai 5 anni gli agenti eziologici virali maggiormente implicati nelle LRI sono gli Ortomyxovirus (Virus influenzali di tipo A e B) $(4,14)$, i Paramyxovirus (Virus parainfluenzali 1-4) (1,3), il Virus Respiratorio Sinciziale (10) ed il Metapneumovirus (17).

Appropriati studi epidemiologici risultano necessari per una programmazione di piani di prevenzione e di sorveglianza epidemiologico-molecolare dei virus respiratori, al fine di individuare eventuali emergenze sanitarie, allestire strategie terapeutiche, preventive e diagnostiche sempre più mirate $\mathrm{e}$, conseguentemente, correlare le manifestazioni cliniche con l'assetto genetico dei virus coinvolti.

Allo scopo di monitorare l'andamento epidemiologico delle ARI in età pediatrica, è stata condotta una sorveglianza virologica attiva degli agenti eziologici più rilevanti, quali virus influenzali ed RSV. Lo studio, condotto da novembre 2005 ad aprile 2005, ha previsto l'arruolamento di bambini di età compresa tra 0 e 10 anni, afferenti presso il Pronto Soccorso Pediatrico e l'UOC Pediatria dell'AO "Ospedale San Carlo Borromeo" di Milano con sintomatologia riconducibile alla definizione di ARI. Gli obiettivi dello studio comprendevano: 1) la diagnosi virologica di infezione da influenzavirus e virus respiratorio sinciziale (RSV) e la successiva identificazione dei sottotipi dei virus influenzali di tipo A; 2) la correlazione dei parametri virologici e di quelli clinici dei soggetti in studio; 3 ) l'analisi epidemiologica della circolazione temporale degli agenti virali indagati durante il periodo in studio.

\section{MATERIALI E METODI}

Pazienti. Da dicembre 2004 ad aprile 2005 sono stati raccolti tamponi faringei da 100 pazienti (51 maschi e 49 femmine), di età compresa tra i 12 giorni e i 10 anni (età media: 19 mesi), con sintomatologia correlabile ad infezione respiratoria acuta, afferenti al Pronto Soccorso Pediatrico ed all'UOC Pediatria dell'AO "Ospedale San Carlo Borromeo" di Milano.

Al momento dell'arruolamento di ciascun paziente, previo consenso informato da parte dei genitori, veniva effettuato un tampone faringeo e veniva compilata una scheda di ingresso riportante: a) indicazioni anagrafiche (età e sesso), b) diagnosi clinica (rinite, bronchite, bronchiolite, polmonite, asma e/o altro), c) sintomatologia (febbre, tosse, secrezioni respiratorie, dispnea e/o altro) e d) informazioni di natura epidemiologica, quali l'eventuale copertura vaccinale contro l'influenza. I tamponi faringei sono stati conservati a $-80^{\circ} \mathrm{C}$, in appropriato terreno di coltura.

Le indagini virologico-molecolari sono state eseguite presso i laboratori della Sezione di Virologia del Dipartimento di Sanità Pubblica, Microbiologia, Virologia dell’Università degli Studi di Milano.

\section{Metodi molecolari}

Estrazione del genoma virale. I campioni biologici sono stati sottoposti ad estrazione di RNA mediante l'utilizzo di un kit commerciale (QIAamp MinElute Virus Spin, Qiagen, Germany).

Retrotrascrizione a $\boldsymbol{c D N A}$. Aliquote da $15 \mu \mathrm{L}$ di RNA estratto sono state sottoposte a retrotrascrizione a DNA complementare (cDNA) mediante utilizzo di random examer primer (Amersham Biosciences, Uppsala-Sweden) a $42^{\circ} \mathrm{C}$ per $1 \mathrm{~h}$ e quindi ad inattivazione enzimatica a $99^{\circ} \mathrm{C}$ per $10^{\prime}$ in termociclatore (GeneAmp® PCR System 9700 - Applied Biosystems, USA).

Multiplex-nested-PCR per Virus influenzali $A, B$ ed $\boldsymbol{R S V}$. La simultanea identificazione dei virus influenzali A e B e del virus respiratorio sinciziale (RSV) è stata condotta mediante una multiplexnested-PCR in house. Sono state sottoposte ad amplificazione regioni geniche, rispettivamente di $169 \mathrm{pb}$ e $310 \mathrm{pb}$, del gene di Matrice $\mathrm{M}$ dei virus influenzali A e B, e di un frammento di 218 $\mathrm{pb}$ del gene codificante la proteina di fusione $\mathrm{F}$ di RSV. La reazione è stata condotta in termociclatore (GeneAmp® PCR System 9700 - Applied Biosystems, USA) alle seguenti condizioni: denaturazione a $95^{\circ} \mathrm{C}$ per $1^{\prime}$, annealing a $52^{\circ} \mathrm{C}$ per 1 ', estensione a $72^{\circ} \mathrm{C}$ per $1^{\prime}$ per 35 cicli nel primo step; denaturazione a $95^{\circ} \mathrm{C}$ per $1^{\prime}$, annealing a $53^{\circ} \mathrm{C}$ per $1^{\prime}$, estensione a $72^{\circ} \mathrm{C}$ per $1^{\prime}$ per 35 cicli nel secondo step di amplificazione.

Multiplex-nested-PCR per la sottotipizzazione del virus influenzale A.Multiplex-nested-PCR 
per la sottotipizzazione del virus influenzale A. I campioni risultati positivi per la presenza di virus influenzale di tipo A sono stati quindi sottoposti a sottotipizzazione mediante multiplex-nested-PCR in house per l'identificazione di frammenti del gene dell'emoagglutinina (HA), rispettivamente di $944 \mathrm{pb}$ per il sottotipo 1 (H1) e di 591 pb per il sottotipo 3 (H3).

L'amplificazione è stata condotta in termociclatore (GeneAmp ${ }^{\circledR}$ PCR System 9700 - Applied Biosystems, USA) alle seguenti condizioni: denaturazione a $94^{\circ} \mathrm{C}$ per 1 ', annealing a $50^{\circ} \mathrm{C}$ per 1 ', estensione a $72^{\circ} \mathrm{C}$ per $1^{\prime}$ per 35 cicli per il primo step; denaturazione a $94^{\circ} \mathrm{C}$ per $1^{\prime}$, annealing a $60^{\circ} \mathrm{C}$ per $1^{\prime}$, estensione a $72^{\circ} \mathrm{C}$ per $1^{\prime}$ per 35 cicli per il secondo step di amplificazione.

I prodotti di amplificazione genica sono stati rilevati in seguito a corsa elettroforetica su gel d'agarosio $2 \%$ e visualizzati ai raggi UV mediante colorazione con bromuro di etidio.

Analisi statistica. Il confronto della frequenza di infezione da RSV e Virus influenzali è stato effettuato mediante test $\chi^{2}$, mentre il confronto tra le età medie dei soggetti con infezioni da RSV e da Influenzavirus mediante test $t$ di Student.

\section{RISULTATI}

Diagnosi virologica. Durante la stagione 20042005, 16 soggetti (16\%), nessuno dei quali vaccinato contro l'influenza nella medesima stagione, sono risultati positivi alla diagnosi virologicomolecolare di infezione da virus influenzali. Di questi, il $75 \%$ è risultato positivo per la presenza di virus A e il $25 \%$ di virus B. Dalle indagini di sottotipizzazione dei 12 campioni biologici di influenza A, $8(66.7 \%)$ sono risultati infetti con il sottotipo A/H3 mentre 4 (33.3\%) con sottotipo A/H1.

Infine, il 14 (14\%) dei tamponi respiratori analizzati ha dato esito positivo per la presenza di sequenze geniche di virus respiratorio sinciziale.

\section{Analisi delle caratteristiche demografiche e cli- niche dei soggetti con infezione da influenzavi- rus ed RSV.}

E stata effettuata una correlazione tra le caratteristiche demografiche e cliniche del gruppo di bambini risultati infetti da virus influenzali o RSV.

I soggetti con infezione da virus influenzale presentavano un'età media di 37.4 mesi (range: 3 mesi -9 anni), più elevata $(\mathrm{p}<0.05)$ rispetto a quella riscontrata tra i soggetti con infezione da RSV (11.7 mesi, range: 12 giorni- 46 mesi). Il $54 \%$ ed il $46 \%$ dei soggetti di sesso femminile è stato riscontrato rispettivamente influenzavirus o RSV positivo.

Come si può rilevare in figura I, si è osservata una differente distribuzione degli eventi clinici segnalati in associazione all'agente eziologico diagno- sticato. È risultata infatti significativamente più elevata la frequenza di bronchiolite $(42.8 \%$ vs $6.2 \% ; \mathrm{p}<0,05)$ e di bronchite $(35.7 \%$ vs $6.2 \%$; $\mathrm{p}<0,05)$ nei casi di infezione da RSV rispetto a quelli di influenza. I casi di polmonite sono risultati numericamente simili in entrambi i tipi di infezione, mentre l'unico caso di asma è stato riscontrato in un bambino di 9 mesi con diagnosi di infezione da virus influenzale $\mathrm{A} / \mathrm{H} 3$.

Le manifestazioni cliniche a carico dell'alto tratto respiratorio (URI) sono risultate più frequentemente associate alle infezioni da virus influenzali.

\section{Distribuzione delle infezioni da influenza e RSV nella stagione influenzale 2004-2005.}

In Italia, il picco di incidenza dell'influenza stagionale è stato segnalato tra la quinta e la sesta settimana del 2005 (6), cioè nelle prime due settimane di febbraio.

Nella coorte pediatrica in studio è stato evidenziato un incremento significativo di casi di infezione da virus influenzali tra la terza e la quarta settimana del 2005, periodo nel quale sono stati identificati il $56.2 \%(9 / 16)$ dei casi (figura II). Più in dettaglio, la prima diagnosi di influenza si è avuta in un campione prelevato nel corso della prima settimana di gennaio e la caratterizzazione virologico-molecolare ha evidenziato la presenza di un ceppo $\mathrm{A} / \mathrm{H} 1$, mentre l'ultimo caso, sostenuto da virus influenzale $\mathrm{A} / \mathrm{H} 3$, è stato registrato in ottava settimana.

Dal punto di vista temporale, la circolazione di virus influenzale $\mathrm{A} / \mathrm{H} 1$ nella popolazione in studio ha preceduto quella dei virus di tipo $\mathrm{A} / \mathrm{H} 3$ e B, identificati a partire dalla seconda settimana del 2005. Inoltre, $\mathrm{i}$ virus di tipo B e A/H1 hanno circolato per periodi più limitati rispetto al tipo $\mathrm{A} / \mathrm{H} 3$ (rispettivamente 3 e 4 settimane $v s 8$ settimane).

Per quanto concerne l'infezione da RSV, nel corso della stagione influenzale indagata non è stato osservato un picco epidemico, sebbene il maggior numero $(21.4 \%, 3 / 14)$ di casi si sia evidenziato in seconda settimana. Il primo caso di infezione da RSV è stato diagnosticato in un soggetto con ARI nella prima settimana del 2005; nel successivo periodo di sorveglianza, la segnalazione di casi è avvenuta in modo costante fino all'ottava settimana, quando è stato registrato l'ultimo caso di infezione respiratoria attribuibile ad RSV (figura II).

\section{CONCLUSIONI}

Indagini epidemiologiche documentano come i soggetti in età pediatrica rappresentino una delle categorie maggiormente a rischio di infezione del tratto respiratorio (15). I virus influenzali ed il virus respiratorio sinciziale sono alcuni dei principali agenti di natura virale responsabili di infezione respiratoria acuta (ARI) (5). 
L'epidemiologia delle ARI è legata sia ad aspetti temporali e spaziali, che alle caratteristiche dell'ospite (12). Una delle peculiarità dei virus respiratori è infatti la stagionalità con cui si presentano nella popolazione umana ed, in particolar modo, nelle fasce d'età più suscettibili, quale quella pediatrica. In particolare, i virus influenzali e l'RSV solitamente circolano nel periodo invernale, con variabilità da regione a regione e da anno ad anno riguardo intensità e durata dei picchi epidemici $(11,12)$. Con l'obiettivo di valutare il grado di coinvolgimento dei virus influenzali e di RSV nelle infezioni respiratorie e di monitorare la loro circolazione nella popolazione suscettibile, è stata condotta una sorveglianza viro-epidemiologica in soggetti in età pediatrica, durante la stagione influenzale 2005-2006.

Lo studio ha rilevato come il $30 \%$ delle sintomatologie respiratorie nella popolazione in studio fosse riconducibile ad infezioni sostenute da uno dei due agenti eziologici considerati.

Sebbene la frequenza di infezione da virus influenzali ed RSV sia risultata paragonabile, le caratteristiche demografiche e cliniche dei soggetti positivi all'indagine virologica presentavano differenze significative. RSV era presente infatti in un elevato numero di infezioni del basso tratto respiratorio (LRI), in particolare nelle bronchioliti e nelle età più precoci. I virus influenzali, invece, risultavano associati principalmente ad infe- zioni dell'alto tratto respiratorio (URI) di soggetti più adulti, compresi principalmente nella fascia di età pre-scolare/scolare. Una possibile spiegazione a queste differenze, sostenuta da studi europei $(18,19)$, potrebbe essere riconducibile alla protezione anticorpale materna, in grado di fornire una buona copertura nei confronti dei virus influenzali (16), ma apparentemente non sufficiente verso l'infezione da RSV (2).

Come atteso sulla scorta dei dati epidemiologici, la circolazione dei due agenti virali si è verificata tra gennaio e febbraio 2005. In particolare, dall'analisi della frequenza di infezioni da influenza ed RSV è stato evidenziato un diverso trend temporale. Infatti, i virus influenzali erano caratterizzati da un andamento epidemico nella popolazione, rappresentato da un picco di casi durante le settimane centrali di gennaio, mentre RSV presentava un trend endemico, monitorato durante l'intero periodo d'indagine.

Nei soggetti con infezione da virus influenzali è stata registrata la presenza dei tre ceppi $(\mathrm{A} / \mathrm{H} 1$, A/H3, B). Questo evento conferma come i soggetti pediatrici rappresentino la fascia di popolazione più suscettibile a diversi agenti eziologici e rivestano quindi il ruolo di "diffusori" (spreaders) degli agenti virali nella popolazione più adulta. Proprio per questa caratteristica, i bambini rappresentano il target principale per la sorveglianza virologica degli isolati virali circolanti finalizzata alla definizione
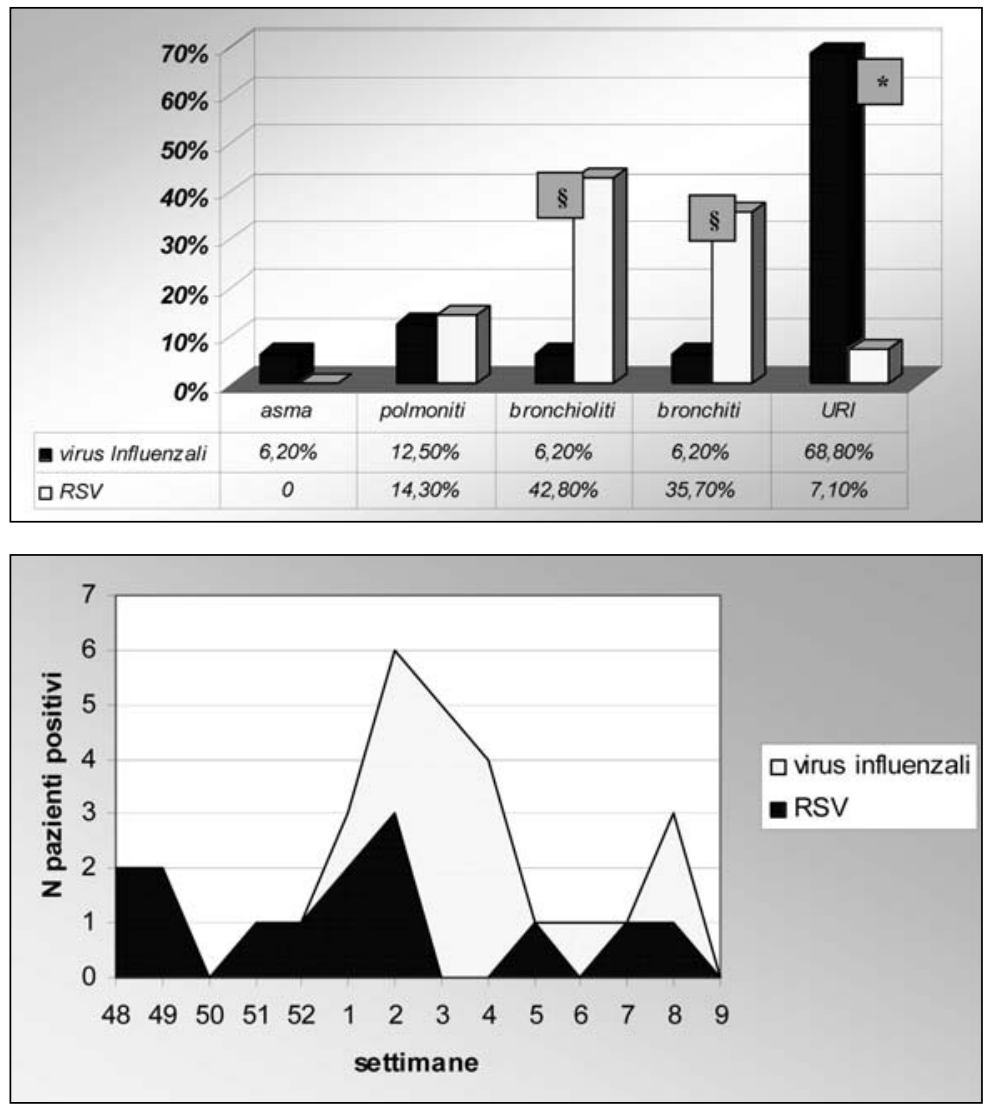

Figura I.

Frequenza delle manifestazioni cliniche diagnosticate nei soggetti con infezioni da virus influenzali ed RSV

$\S p<0.05$; bronchioliti (42.8\% vs 6.2\%) e bronchiti (35.7\% vs 6.2\%) nei casi di infezione da RSV vs casi di infezione da virus influenzali; * $p$ 0.05; URI (68.8\% vs $7.1 \%$ ) nei casi di infezione da virus influenzali vs casi di infezione da RSV.

Figura II.

Distribuzione delle infezioni da virus influenzali ed RSV nella stagione influenzale 2004-2005 
della annuale composizione dei vaccini $(11,12)$. In conclusione, durante la stagione influenzale 2004-2005, i virus influenzali e l'RSV hanno rappresentato due degli agenti virali responsabili di elevata morbosità in soggetti in età pediatrica.

Il nostro studio valorizza l'importanza di una sorveglianza attiva delle virosi respiratorie nei casi di richiesta di ospedalizzazione per infezioni del tratto respiratorio nelle fasce d'età più giovani. L'approfondimento delle conoscenze riguardanti le caratteristiche demografiche del paziente, le proprietà antigeniche degli agenti virali e la loro stagionalità risultano elementi integranti la valutazione degli episodi clinici.

\section{BIBLIOGRAFIA}

1. Bellini WJ, Rota PA, Anderson LJ. Paramyxoviruses in: Microbiology and microbial infections (Virology), L. Collier, A. Balows, M. Sussmann - Arnold Ed. 1998; 23: 435-52.

2. Bulkow LR, Singleton RJ, Karron RA, Harrison LH, Group ARS. Risk factors for severe respiratory syncytial virus infection among Alaska native children. Pediatrics 2002; 109: 210-6.

3. Chanock RM, McIntosh K. Parainfluenza Viruses in: Virology, B. N. Fields - Raven Press, New York, 1990; 35: 963-84.

4. Cox NJ, Kawaoka Y. Orthomyxoviruses: influenza in: Microbiology and microbial infections (Virology), L. Collier, A. Balows, M. Sussmann - Arnold Ed, 1998; 22: 386-418.

5. Fleming DM, Pannell RS, Elliot AJ, Cross KW. Respiratory illness associated with influenza and respiratory syncytial virus infection. Arch Dis Child 2005; 90: 741-6.

6. http//www.influnet.it

7. http//www.who.int

8. Klig JE. Current challenges in lower respiratory infections in children. Current Opinion in Pediatrics 2004; 16: 107-12.

9. Mackie PL. The classification of viruses infecting the respiratory tract. Ped. Resp. Rew. 2003; 4: 84-90.

10. McIntosh K, Chanock RM. Respiratory Syncytial Virus in: Virology, B. N. Fields - Raven Press - New York, 1990; 38:1045-66.

11. Monto AS. Epidemiology of viral respiratory infections. AJM 2002; 112(6A): 4S-12S.

12. Monto AS. Occurence of respiratory virus: time, place and person. Pediatr Infect Dis J 2004; 23(1): S58-S64.

13. Moroni M, D'Arminio Monforte A, Milazzo L. Infezioni dell'apparato respiratorio in "Malattie Infettive", M. Moroni, R. Esposito, F. De Lalla, Masson Ed. - Milano, 2003; 9: 355-80

14. Nicholson KG, Wood JM, Zambon M. Influenza. Lancet 2003; 362: 1733-45.

15. Programme for the Control of Acute Respiratory Infections. Interim Programme Report 1992. Geneva: WHO; 1993 (WHO/ARI/93.25).

16. Reuman PD, Ayoub EM, Small PA. Effect of passive maternal antibody on influenza illness in children: a prospective study of influenza $\mathrm{A}$ in mother-infant pairs. Pediatr Infect Dis J 1987;6:398-403.

17. Van Den Hoogen BG, De Jong JC, Groen J, et al. A newly discovered human pneumovirus isolated from young children with respiratory tract disease. Nat.
Med. 2001; 7: 719-24.

18. Weigl JAI, Puppe W, Schmitt HJ. The incidence of influenza-associated hospitalizations in children in Germany. Epidemiol Infect 2002;129:525-33.

19. Winter GF, Hallam NF, Hargreaves FD, Molyneaux PJ, Burns, SM, Inglis JM. Respiratory viruses in a hospitalized paediatric population in Edimburgh 1985-1994. J Infect 1996; 33: 207-11. 